\title{
Axial particle positioning by wavefront parameterization using Chebyshev polynomials and off-axis digital holography
}

\author{
Johan Öhman \& Mikael Sjödahl \\ Experimental Mechanics, Luleå University of Technology, SE-971 87 Luleå, Sweden \\ johan.ohman@ltu.se
}

\begin{abstract}
A particle can be axially positioned where its scattered light has a plane wavefront. The phase anomaly compared to a plane wave is fitted to 3D Chebyshev polynomial, where coefficients correspond to the axial position.

OCIS codes: (8090.1995) Digital holography; (090.1760) Computer holography; (290.5850) Scattering, particles
\end{abstract}

\section{Introduction}

Axial particle positioning in digital-holography is a non-trivial task since the axial resolution is severely worse than the lateral one for moderate numerical apertures [1]. A metric is therefore most commonly applied to determine the axial position. Reported metrics have been based on intensity [2], intensity gradients [3] or a combination of both [4], complex metrics have also been reported [5,6]. We have in a previous paper showed that the axial particle position can be found by tracking the wavefront curvature [7]. In that paper the curvature was estimated in each reconstructed plane by computing the phase gradients using the finite difference method. This method is, however, quite sensitive to noise since the finite difference operator essentially acts as a high pass filter. In this paper we parameterize the unwrapped phase anomaly response using three dimensional Chebyshev polynomials. In addition the intensity is used as weights in the parameterization so that high intensity voxels influence the estimation more than voxels with lower intensity. From the Chebyshev parameters it is possible to extract information about the axial position of the particle. By parameterization the wavefront curvature estimation becomes more robust.

\section{Theory}

Micrometer sized particle will scatter light according to the Lorentz-Mie theory, for a full description we refer the reader to [8]. In the imaging process a limited set of scattering directions are used to form the spatial frequency content in the front focus plane according to the Lorentz-Mie theory. The scattered light is simulated to be imaged by a bi-telecentric setup, described in [7]. Then the field present on the detector $\mathrm{U}_{\mathrm{d}}$ becomes:

$$
U_{d}\left(x^{\prime}, y^{\prime}\right)=\sqrt{\frac{1}{M}} U_{i}\left(-\frac{x}{M},-\frac{y}{M}\right),
$$

where $M$ is the magnification of the imaging system and $U_{i}$ is the field in the front focus plane. To form a hologram on the detector an off-axis reference wave, $R$, is added and the total intensity on the detector, $I\left(x^{\prime}, y^{\prime}\right)$ is then given by:

$$
I\left(x^{\prime}, y^{\prime}\right)=\left|U_{d} R\right|^{2}=\left|U_{d}\right|^{2}+|R|^{2}+U_{d}{ }^{*} R+U_{d} R^{*} .
$$

Since the reference wave is off-axis it is possible to filter out the term $U_{d} R^{*}$ in the Fourier domain. If the reference light incident on the detector is a plane wave the term $R^{*}$ becomes unity so that $U_{d}$ can be retrieved directly. By knowing the complex amplitude $U_{d}$ of the scattered light it is possible to refocus it and hence reconstruct a 3D volume around the imaged scene. The angular spectrum method is used to perform this reconstruction. [9] The phase in the 3D volume is used to determine the axial position of the particle. The first step in doing so is to compute the phase anomaly compared to a plane wave, i.e. to subtract the phase contribution corresponding to a plane wave, the phase anomaly then becomes:

$$
\varphi_{a}(x, y, z)=\varphi(x, y, z)-k z \text {. }
$$

The direct phase is not used since it is hard to resolve the phase efficiently since the $k z$ term varies rapidly along the optical axis. The phase anomaly is fitted to a three dimensional Chebyshev polynomial of first kind. Chebyshev polynomials are orthogonal, a more detailed description is provided in [10]. A three-dimensional Chebyshev polynomial expansion of degree $n$ is described as:

$$
\psi(x, y, z)=\sum_{i=0}^{n} \sum_{j=0}^{n} \sum_{k=0}^{n} a_{i j k} T_{i}(x) T_{j}(y) T_{k}(z),
$$


where $T_{i}$ is the one dimensional Chebyshev polynomial of first kind of degree $i$, and $a_{i j k}$ is the associated polynomial coefficients. This expression can be rewritten in matrix notion as the scalar product:

$$
\psi(x, y, z)=\boldsymbol{T}(x, y, z) \cdot \bar{a} .
$$

Since the Chebyshev polynomials only are defined on the domain $[-1,1]$ the coordinates needs to be rescaled to this domain. The fitting problem is then solved by finding the solution to:

$$
\hat{\mathrm{a}}_{i j k}=\underset{a}{\operatorname{argmin}} \sum W\left(\varphi_{a}-T(x, y, z) \cdot \bar{a}\right)^{2},
$$

where $W$ are the weights that influence how much each data point influence the estimation. Eq.(8) is an optimization problem, the solution to the weighted lest squares problem becomes:

$$
\hat{\mathrm{a}}_{i j k}=\left(\boldsymbol{T}^{T} W \boldsymbol{T}\right)^{-1} \boldsymbol{T}^{T} W \varphi_{a}
$$

The polynomial coefficient vector contains $n^{3}$ coefficients that hold information regarding the position of the particle. The volume in which the estimation is done is decided with regards to the maximum intensity of the particle.

\section{Method and Results}

In our previous paper [7], we concluded that the plane where phase gradients perpendicular to the scattering plane are zero is the axial location of the particle. This is equivalent to finding a Chebyshev polynomial fit that does not depend on pure terms perpendicular to the scattering plane. If the scattering plan is spanned by the $x$ and $z$ axis, polynomials that only contains y-terms needs to have a zero coefficient. The axial location is found by fitting the Chebyshev polynomials to axially shifted versions of the phase anomaly. The shift where these y-dependent coefficients are zero is then the location of the particle. Since an offset in the Fourier filtering of the hologram corresponds to a linear phase shift only the coefficient $\hat{a}_{020}$, which corresponds to the $y^{2}$ term is tracked in order to be invariant to these linear phase shifts. An example of this response in the coefficient $\hat{a}_{020}$ is presented in Fig.1a for different shifts. Using simulations a large set of particles is individually parameterized to Chebyshev polynomials up to degree of $n=2$. In the simulations and later in experimental measurements an imaging system with magnification $M=1.33$, numerical aperture $N A=0.025$, pixel pitch $d=3.45 \mu \mathrm{m}$ and wavelength $\lambda=532 \mathrm{~nm}$ is used. First segmentation is performed so that individual particles can be distinguished. Eq.(8) is solved for each segmented particle and the weights, $W$, are chosen to be the intensity in the corresponding voxel. By doing so only phase content where light from the particle is present is used in the estimation. Contour plots from simulations for both the measured phase anomaly and the corresponding estimated Chebyshev polynomials are showed in Fig.1b and Fig.1c respectively. The estimated data from the Chebyshev polynomials captures the behavior and curvature of the measured data, the phase anomaly, well.
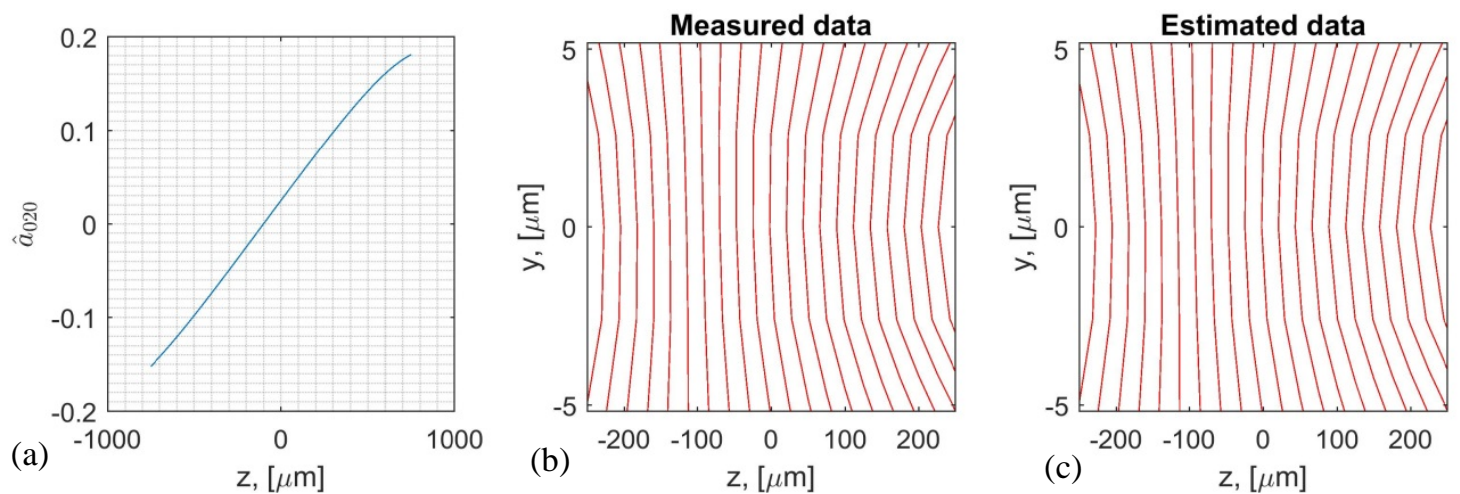

Fig. 1. (a) shows the evaluation of the coefficient â ${ }_{020}$ for different shifts. The coefficient becomes zero at the axial location of the particle. (b) and (c) shows contour plots of cross sections in the measured phase anomaly data and estimated phase anomaly data from the fitted polynomial, respectively.

The axial position is then found by finding the shift that corresponds to $\hat{a}_{020}=0$. The result from the simulation is an average error of $0.4 \mu \mathrm{m}$ and a standard deviation of $8.0 \mu \mathrm{m}$ for a peak to peak noise ratio of three percent. Outliers are removed before calculating these values. Comparing this to the similar method that utilized finite differences a decrease in standard deviation can be noted for increasing noise levels. Standard deviations reported previously using finite differences ranged from 2.6-39.5 $\mu \mathrm{m}$ for noise ratios of $0-4$ percent [7]. By utilizing Chebyshev polynomial coefficients instead the standard deviation ranged from 2.6-9.1 $\mu \mathrm{m}$ for the same noise ratios. This 
increased accuracy can be explained by the fact that the noise is assumed to be zero mean and by fitting a numerical model to the data instead of performing finite differences noise in individual pixels do not have the same impact. A simple experiment is performed to determine that the parameterization was applicable on experimental data as well. The experimental setup has the same properties as the simulated one, shown in Fig.2. The light source is a $\mathrm{Nd}$ :Yag laser with wavelength, $\lambda=532 \mathrm{~nm}$, the beam is divided by a 50/50 beam splitter. One part is expanded by the lenses $L 1$ and $L 2$ to illuminate a larger area on the sample. The other part is focused into a polarization maintaining fiber using a mirror and fiber coupling. The object consists of micrometer particles molded into silicone; the particles have an average radius of $5 \mu \mathrm{m}$. The sample is imaged by a bi-telecentric imaging system consisting of lenses $L 3, L 4$ and the aperture $A$. The system has a magnification of $M=1.33$. The end of the fiber is connected to the aperture to enable off-axis holographic recording. From recorded holograms a reconstructed volume is formed. The positioning thereafter followed the same procedure as in the simulation. The object is stationary and particles are tracked over several recordings. The mean for each particle is computed and subtracted to compute the error. These errors are then comparable regarding of which particle they belonged to. A histogram of the errors is presented in Fi-g. 2.(b). From these values the standard deviation is calculated to be $12.8 \mu \mathrm{m}$, after filtering out outliers.
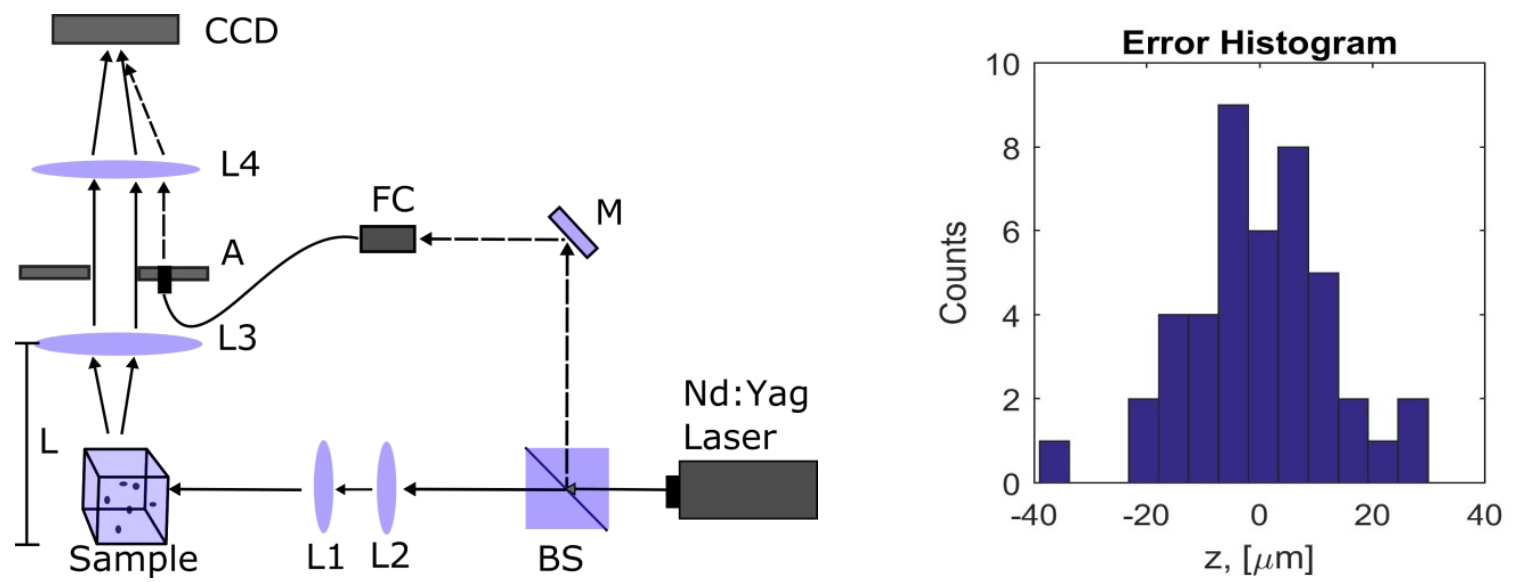

Fig. 2. (a) shows the setup used. Lenses are indicated with L, beam splitter with BS, mirror with M, aperture with A and fiber coupling with FC. (b) shows the histogram of experimentally estimated errors.

Comparing this to the simulated values it is obvious that the experimental data is less accurate than the simulated ones. It is however an improvement compared to the result obtained using finite differences where the experimentally obtained standard deviation was estimated to be $17.9 \mu \mathrm{m}$ [7].

What we can conclude is that fitting the measured phase anomaly to a three dimensional Chebyshev polynomial is a more robust method of tracking wavefront curvature than estimating the in plane phase gradients using finite differentials. This is because noise in individual pixels is not affecting the estimation in the same extent. From the Chebyshev polynomial coefficients it is possible to determine the axial particle position tracking a selected coefficient that corresponds to the $\mathrm{y}^{2}$-polynomial.

\section{References}

[1] M. Born and E. Wolf, Principles of Optics: Electromagnetic Theory of Propagation, Interference and Diffraction of Light, 7th ed. (Cambridge University, 1999).

[2] Y. S. Bae, J. I. Song, and D. Y. Kim, "Volumetric reconstruction of Brownian motion of a micrometer-size bead in water,” Opt. Commun. 309, 291-297 (2013).

[3] L. Wilson and R. Zhang, "3D Localization of weak scatterers in digital holographic microscopy using Rayleigh-Sommerfield backpropagation,” Opt. Express 20, 16735-16744 (2012).

[4] J. K. Abrantes, M. Stanislas, S. Coudert, and L. F. A. Azevedo, “Digital microscopic holography for micrometer particles in air,” Appl. Opt. 52, A397-A409 (2013).

[5] G. Pan and H. Meng, "Digital holography of particle fields: reconstruction by use of complex amplitude,” Appl. Opt. 42, 827-833 (2003).

[6] J. de Jong and H. Meng, "Digital holographic particle validation via complex wave,” Appl. Opt. 46, 7652-7661 (2007).

[7] J. Öhman and M. Sjödahl, "Off-axis digital holographic particle positioning based on polarization-sensitive wavefront curvature estimation," Appl. Opt. 55, 7503-7510 (2016)

[8]C. F. Bohren and D. R. Huffman, Absorption and Scattering of Light by Small Particles (Wiley, 1998).

[9] J. W. Goodman, Introduction to Fourier Optics, 2nd ed. (McGraw-Hill, 1996).

[10] Abramowitz, Milton \& Stegun, Irene A. (red.), Handbook of mathematical functions: with formulas, graphs, and mathematical tables, (Dover, New York, 1972) 